\title{
Tunneling and the Vacuum Zero-Point Radiation
}

\author{
H. M. França, G. G. Gomes, and R. L. Parra \\ Instituto de Física, Universidade de São Paulo \\ C.P. 66318, 05315-970 São Paulo, SP, Brazil
}

Received on 29 September, 2006

\begin{abstract}
We make a brief review of the Kramers escape rate theory for the probabilistic motion of a particle in a potential well $U(x)$, and under the influence of classical fluctuation forces. The Kramers theory is extended in order to take into account the action of the thermal and zero-point random electromagnetic fields on a charged particle. The result is physically relevant because we get a non null escape rate over the potential barrier at low temperatures $(T \rightarrow 0)$. It is found that, even if the mean energy is much smaller than the barrier height, the classical particle can escape from the potential well due to the action of the zero-point fluctuating fields. These stochastic effects can be used to give a classical interpretation to some quantum tunneling phenomena. Relevant experimental data are used to illustrate the theoretical results.
\end{abstract}

Keywords: Zero-point fluctuations; Tunneling; Foundations of quantum mechanics

\section{INTRODUCTION}

One of the most useful contributions to our understanding of the stochastic processes theory is the study of escape rates over a potential barrier. The theoretical approach, first proposed by Kramers [1], has many applications in chemistry kinetics, diffusion in solids, nucleation [2], and other phenomena [3]. The essential structure of the escape process is that the bounded particle is under the action of three types of forces: a deterministic nonlinear force with at least one metastable region, a fluctuating force whose action is capable of pushing the particle out of the metastable region, and a dissipative force which inevitably accompanies the fluctuations.

In this work we describe the escape rates of a particular model: a classical charged particle moving in the metastable potential shown in the Fig. 1, and under the influence of the fluctuating electromagnetic radiation forces commonly used in Stochastic Electrodynamics (SED) [4, 5].

The fluctuating fields postulated in SED are classical random fields, with zero mean but nonzero higher moments. The spectral distribution of this radiation can be expressed as a sum of two terms

$$
\rho(\omega, T)=\frac{\omega^{2}}{\pi^{2} c^{3}}\left[\frac{\hbar \omega}{2}+\frac{\hbar \omega}{e^{\hbar \omega / k_{B} T}-1}\right]
$$

The first term is the zero-point radiation contribution to the spectral distribution. It is independent of the temperature and is Lorentz invariant. The second term in (1) is the blackbody radiation spectral distribution, responsible for the temperature effects on the system.

The zero-point radiation (first term in (1)) has a mean energy $\hbar \omega / 2$ associated with each mode of the electromagnetic fields, and is responsible for the most important features of SED.

With this zero-point radiation postulated, several phenomena associated with the quantum behavior of the microscopic world can be explained on classical grounds. Many interesting examples can be found in the reviews [5-8].

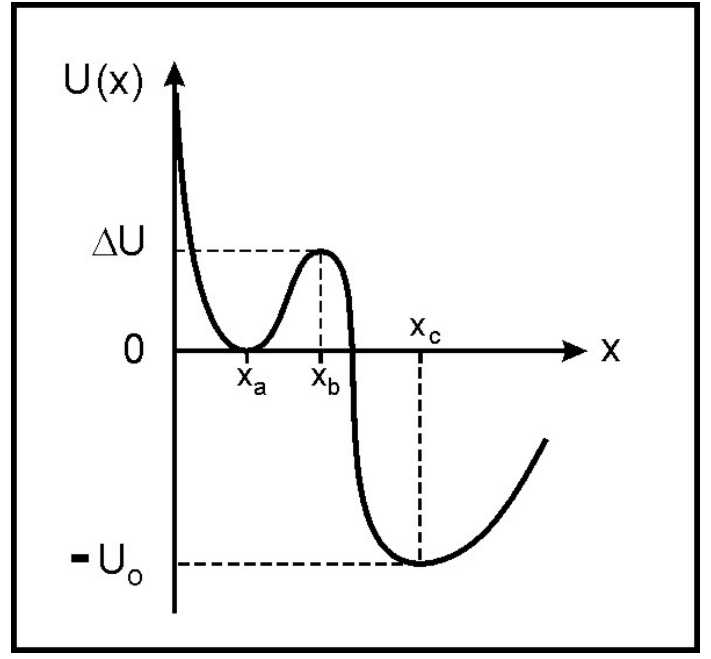

FIG. 1: Metastable potential with a barrier height $\Delta U=U\left(x_{b}\right)-$ $U\left(x_{a}\right)$, a local minimum $x_{a}$ and a local maximum $x_{b}$ (top of the barrier). The second minimum at $x_{c}$ has a potential energy $U\left(x_{c}\right)=-U_{0}$ with $U_{0} \gg \Delta U$.

\section{HARMONIC OSCILLATOR WITH STOCHASTIC RADIATION}

The potential $U(x)$ will be approximated by a harmonic oscillator in the region of the potential well $\left(x \approx x_{a}\right)$ so that (see Fig. 1)

$$
U(x) \simeq U\left(x_{a}\right)+\frac{1}{2} m \omega_{a}^{2}\left(x-x_{a}\right)^{2},
$$

where $\omega_{a}$ is the natural frequency of the oscillator.

The dynamical behavior of a harmonically bounded charged particle has been extensively studied in the context of classical SED. It is found that the zero-point radiation maintains the stability of this system. We shall use the statistical properties of the harmonic oscillator in order to understand, classically, the escape rate at very low temperatures. We give below a brief review of the harmonic motion under the action of the random electric fields characteristic of SED. 
The nonrelativistic motion of the charged particle (charge $e$ and mass $m$ ) near the bottom of the potential well (see Fig. 1) is governed by the equation

$$
m \ddot{\xi}=-m \omega_{a}^{2} \xi+\frac{2 e^{2}}{3 c^{3}} \dddot{\xi}+e E_{x}(t),
$$

where $\xi=x-x_{a}$, and $E_{x}(t)$ is the $x$ component of the random electric field. The term proportional to $\dddot{\xi}$ is the radiation reaction force. The electric field is such that $\left\langle E_{x}(t)\right\rangle=0$ and

$$
\begin{aligned}
& \left\langle E_{x}(t) E_{x}(0)\right\rangle= \\
& \quad \frac{4 \pi}{3} \int_{0}^{\infty} d \omega \rho(\omega, T) \cos (\omega t),
\end{aligned}
$$

where the spectral distribution $\rho(\omega, T)$ was introduced in the equation (1). The radiation reaction force can be approximated by [9]

$$
\frac{2 e^{2}}{3 c^{3}} \dddot{\xi} \simeq-m \gamma \dot{\xi},
$$

where $\gamma=2 e^{2} \omega_{a}^{2} / 3 m c^{3}$. Moreover it is verified that $\gamma \ll$ $\omega_{a}$. According to these approximations one can show that the average energy $\left\langle\varepsilon_{a}\right\rangle$ of the oscillating charge is such that

$$
\begin{gathered}
\left\langle\varepsilon_{a}\right\rangle=\frac{1}{2} m\left\langle\dot{\xi}^{2}\right\rangle+\frac{1}{2} m \omega_{a}^{2}\left\langle\xi^{2}\right\rangle= \\
\frac{\hbar \omega_{a}}{2} \operatorname{coth}\left(\frac{\hbar \omega_{a}}{2 k_{B} T}\right) \equiv D(T),
\end{gathered}
$$

where we have introduced the function $D(T)$ in order to simplify our notation. Notice that the average energy depends on the temperature and on the oscillatory frequency $\omega_{a}$.

The result (6) is well known [5]. The average energy $\left\langle\varepsilon_{a}\right\rangle$ becomes equal to $k_{B} T$ in the high temperature limit $\left(k_{B} T \gg\right.$ $\left.\hbar \omega_{a}\right)$, and is non zero when $T=0$. Actually $D(T) \rightarrow \hbar \omega_{a} / 2$ as $T \rightarrow 0$. Notice that $D(T)$ depends on $\hbar$. We can show that the Planck constant comes from the intensity of the zero-point field $E_{x}(t)$ that appears in (3). We recall that $\hbar \omega_{a} / 2$ is the value of the ground state energy of the harmonic oscillator in quantum mechanics. This result, obtained within the realm of SED, differs from the usual null result of ordinary classical physics because the zero-point fluctuations are taken into account.

It is quite natural to use the average energy (6) in the calculation of the Kramers escape rate of a potential well. We shall see that the consequence of the new form of the average energy is a non-vanishing escape rate even if $T \rightarrow 0$. Most authors do not mention the classical zero-point fluctuations and use the quantum mechanical formalism to interpret the non null escape rate as a tunneling through the classical forbidden region of the barrier. We shall see that the zero-point fluctuations allow the escape over the potential barrier even if the mean energy of the particle inside the barrier is much less than $\Delta U=U\left(x_{b}\right)-U\left(x_{a}\right)$. In the classical mechanics context the escape would be impossible without the action of the zeropoint fluctuations. For simplicity we shall take $U\left(x_{a}\right)=0$ in what follows.

\section{THE ESCAPE RATE OVER THE POTENTIAL BARRIER $\Delta U$}

We shall use the approach of Chandrasekhar [10], based on the Kramers theory. The physical system considered by Chandrasekhar is a particle moving under the influence of a fluctuating force, and a potential $U(x)$ that has a metastable region (see Fig. 1). The motion of the particle is governed by a Langevin type equation

$$
m \ddot{x}=-m \gamma \dot{x}-U^{\prime}(x)+F(t),
$$

where $-m \gamma \dot{x}$ is the dissipative force and $F(t)$ is the fluctuating force which is characterized by the average $\langle F(t)\rangle=0$. The average energy of the particle within the potential well, that is $x<x_{b}$, is assumed to be given by

$$
\left\langle\frac{m}{2} \dot{x}^{2}+U(x)\right\rangle=k_{B} T,
$$

in the high temperature limit.

It is possible to show that the Langevin equation (7) leads to a phase space Fokker-Planck equation given by [10]

$$
\begin{gathered}
\frac{\partial W}{\partial t}+\frac{p}{m} \frac{\partial W}{\partial x}-U^{\prime}(x) \frac{\partial W}{\partial p}= \\
\gamma W+\gamma p \frac{\partial W}{\partial p}+m \gamma D(T) \frac{\partial^{2} W}{\partial p^{2}}
\end{gathered}
$$

where $W=W(x, p, t)$ is the probability distribution in phase space.

Notice that the left hand side of the above expression is equivalent to the Liouville equation, and the right hand side appears as a consequence of the fluctuating and dissipation forces. This expression is valid for low temperatures, because we included $D(T)=\frac{\hbar \omega_{a}}{2} \operatorname{coth}\left(\frac{\hbar \omega_{a}}{2 k_{B} T}\right)$ (see equation (6)), instead of the factor $k_{B} T$ as is usually done. We shall see that the equation (9) will allow us to give an accurate description of the escape rate at low temperatures.

In the Kramers theory, two quantities are essential to calculate the escape rate. One is the probability $P(t)$ of finding the particle inside the potential well. This probability can be obtained from the phase space distribution, namely

$$
P(t)=\int_{-\infty}^{\infty} d p \int_{-\infty}^{x_{b}} d x W(x, p, t)
$$

The other important quantity is the diffusion current, $j\left(x_{b}\right)$, across the top of the potential barrier. The diffusion current in an arbitrary position $x$ is defined by

$$
j(x, t) \equiv \int_{-\infty}^{\infty} d p \frac{p}{m} W(x, p, t) .
$$

Using (9), (10) and (11) one can show that 


$$
\begin{gathered}
\frac{\partial P(t)}{\partial t}=-\int_{-\infty}^{\infty} d p \frac{p}{m} W\left(x_{b}, p, t\right) \\
=-j\left(x_{b}\right) e^{-\kappa T} .
\end{gathered}
$$

The escape rate $\kappa$, regarded as the decay factor of the probability $P(t)$, can be defined by the equation

$$
\frac{\partial P(t)}{\partial t}=-\kappa P(t)
$$

The solution of the above equation is

$$
P(t)=P_{0} e^{-\mathrm{k} t},
$$

where $P_{0}$ is a normalization constant. On the other hand, consistently with the equations (12) and (13), one can define the escape rate as

$$
\kappa \equiv \frac{j\left(x_{b}\right)}{P_{0}}
$$

A long calculation considered in Ref [14], in the low friction limit,

$$
\frac{\gamma}{\omega_{a}}=\frac{e^{2}}{\hbar c} \frac{\hbar \omega_{a}}{m c^{2}} \ll 1
$$

leads to the simple formula

$$
\kappa(T)=\frac{\omega_{a}}{2 \pi} \exp \left(-\frac{\Delta U}{\frac{\hbar \omega_{a}}{2} \operatorname{coth}\left(\frac{\hbar \omega_{a}}{2 k_{B} T}\right)}\right) .
$$

It is very important to remark that, in this equation, the escape rate depends on the potential height $\Delta U$, and on the parameters characterizing the particle motion inside the barrier $\left(x \approx x_{a}\right)$. These parameters are the frequency $\omega_{a}$ and the average energy $\left\langle\varepsilon_{a}\right\rangle=D(T)=\frac{\hbar \omega_{a}}{2} \operatorname{coth}\left(\frac{\hbar \omega_{a}}{2 k_{B} T}\right)$. We recall that $\left\langle\varepsilon_{a}\right\rangle=k_{B} T$ when the temperature is high enough.

\section{COMPARISON WITH EXPERIMENTAL DATA AND CONCLUSION}

In order to illustrate, in a quantitative manner, the great analogy between the quantum tunneling description and our classical stochastic escape rate calculation, the experimental results of Alberding et al. [11] will be used.

According to Alberding et al., the carbon monoxide $C O$ is bounded to the beta-chain of hemoglobin $(\beta H b)$ from which it can be separated with a LASER. The rate of recombination can be obtained experimentally. The fraction $N(t)$ of the $C O$ molecules that have not been recombined with the $\beta H b$ is measured as a function of time. Then, the time $\tau$, necessary to reduce $N(t)$ to $75 \%$ of its original value, is determined. It is assumed that this recombination is a passage of the $C O$ molecule through the potential barrier $\Delta U\left(x \approx x_{a} \rightarrow x \approx x_{c}\right)$ and a good estimate of the escape rate is $\kappa=1 / \tau$ (see our Fig. 1 and the Fig. 1a of Alberding et al. [11]). This experimental procedure can be repeated for different temperatures $T$. The result for $\kappa(T)$ obtained by Alberding et al. is indicated by the experimental points (black dots) in the Fig. 2. We see that these experimental data are very well described by the formula (17).

We have adjusted the values of $\omega_{a}$ and $\Delta U$ so that the experimental data and the formula (17) are in good agreement. The values obtained are

$$
\begin{aligned}
\frac{\hbar \omega_{a}}{2} & =2.53 \cdot 10^{-3} \mathrm{eV}, \\
\Delta U & =6.68 \cdot 10^{-2} \mathrm{eV} .
\end{aligned}
$$

We conclude that the $C O$ particle can escape from the potential well at $T \rightarrow 0$, despite the fact that the barrier height $\Delta U$ is much bigger than the particle mean energy $\hbar \omega_{a} / 2$ inside the well $\left(2 \Delta U / \hbar \omega_{a} \approx 26\right)$.

We would like to make some observation concerned with the role of the vacuum electromagnetic zero-point fluctuations within the Kramers equation and its relation with the Schrödinger equation for describing tunneling phenomena. According to the extension of the Kramers theory presented here, the escape rate at low temperature is $\kappa(T \simeq$ $0) \propto \exp \left(-\frac{2 \Delta U}{\hbar \omega_{a}}\right)$. The quantum calculation based on the Schrödinger equation is more cumbersome if we want to obtain $\kappa_{Q M}(T \simeq 0)$ from the value $\kappa_{Q M}(T)$ obtained at an arbitrary temperature $T$ (see [11] for instance).

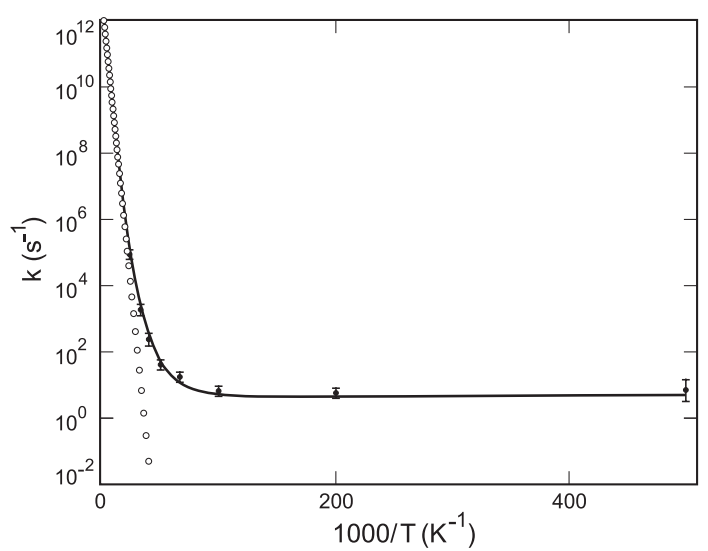

FIG. 2: Experimental data (black dots) for the escape rate associated with the CO migration to a separated $\beta$ chain of hemoglobin. The solid line is our theoretical result (see formula (17)). The open circles correspond to the escape rate without the zero-point fluctuations (Arrhenius formula).

This difficulty arises because the quantum system is in a heat bath, and because the potential $U(x)$ is complicated enough (see Fig. 1). However, for a more simple potential, like the double well harmonic oscillator described by $\mathrm{E}$. Merzbacher [12], it is possible to show that $\kappa_{Q M}(T=0) \propto$ $\exp \left(-\frac{2 \Delta U}{\hbar \omega_{a}}\right)$. This result is in semiquantitative agreement 
with our classical stochastic calculation based on the Kramers theory with zero-point radiation. Finally, we would like to say that the direct inclusion of zero-point and thermal fluctuations into the Schrödinger equation [13] is a very delicate problem that is out of the scope of the present work.

\section{Acknowledgements}

We thank the financial support from Fundação de Amparo à
Pesquisa do Estado de São Paulo (FAPESP) and Conselho Nacional de Desenvolvimento Científico e Tecnológico (CNPq-Brazil). We also thank Prof. C. P. Malta and Prof. E. Santos for valuable comments.
[1] H. A. Kramers, Physica 7, 284 (1940).

[2] P. Hänggi, P. Talkner, and M. Borkovec, Rev. Mod. Phys. 62, $251(1990)$

[3] T. W. Marshall and E. Santos, "Semiclassical treatment of macroscopic quantum relaxation", Anales de Fisica 91, 49 (1995).

[4] T. W. Marshall, "Random electrodynamics", Proc. Royal Soc. London A 276, 475 (1963).

[5] T. H. Boyer, "Random electrodynamics: The theory of classical electrodynamics with classical electromagnetic zero-point radiation", Phys. Rev. D 11, 790 (1975).

[6] T. H. Boyer, "A Brief Survey of Stochastic Electrodynamics" in: Foundations of Radiation Theory and Quantum Electrodynamics, A. O. Barut, ed. (Plenum, New York, 1980), pg. 49.

[7] L. de la Peña and A. M. Cetto, The Quantum Dice. An Introduction to the Stochastic Electrodynamics (Kluwer Academic,
Dordrecht, 1996).

[8] P. W. Milonni, Phys. Rep. 25, 1 (1976).

[9] J. D. Jackson, Classical Electrodynamics, 2nd edn. (Wiley, New York, 1975), chapters 9 and 17.

[10] S. Chandrasekhar, Rev. Mod. Phys. 15, 1 (1943).

[11] N. Alberding, R. H. Austin, K. W. Beeson, S. S. Chan, L. Eisenstein, H. Frauenfelder, and T. M. Nordlund, Science 192, 1002 (1976).

[12] E. Merzbacher, Quantum Mechanics (Wiley, New York, 1970). See in particular the tunneling associated with the ammonia molecule $\left(\mathrm{NH}_{3}\right)$ (section 5, equation (5.63)).

[13] A. J. Faria, H. M. França, C. P. Malta, and R. C. Sponchiado, Phys. Lett. A 305, 322 (2002).

[14] A. J. Faria, H. M. França, and R. C. Sponchiado, Found. Phys. 36, 307 (2006). 